\section{Original} article

\author{
Department of \\ Sexually Transmitted \\ Diseases, Royal Free \\ and University College \\ Medical School, \\ London, UK \\ K A Fenton \\ A Copas \\ A M Johnson \\ The National Centre \\ for Social Research, \\ London \\ G Elam \\ B Erens \\ J Field
}

London School of Hygiene and Tropical Medicine, London

$\mathrm{K}$ Mitchell

$\mathrm{K}$ Wellings

Department of Microbiology, University College Hospital, London C Carder

G Ridgway

The Public Health Laboratory Service, Communicable Disease Surveillance Centre

K A Fenton

Correspondence to: Dr Kevin Fenton, Department of Sexually Transmitted Diseases, Royal Free and University College Medical School, Mortimer Market Centre, off Capper Street, London WC1E 6AU, UK

kfenton@gum.ucl.ac.uk

Accepted for publication 2 February 2001

website

extra

A table detailing response rates appears on the STI website.

www.sextransinf.com

\title{
The acceptability of urinary LCR testing for Chlamydia trachomatis among participants in a probability sample survey of sexual attitudes and lifestyles
} Kevin A Fenton, Andrew Copas, Kirstin Mitchell, Gillian Elam, Caroline Carder,
Geoff Ridgway, Kaye Wellings, Bob Erens, Julia Field, Anne M Johnson

Objectives: To examine the factors that influence respondents' willingness to participate in urinary testing for Chlamydia trachomatis in a general population feasibility survey of sexual attitudes and lifestyles.

Methods: 199 sexually experienced, 18-44 year old participants, recruited as part of a larger $(\mathrm{n}=901)$ methodological study of sexual attitudes and lifestyles, were invited to provide a urine sample for chlamydial infection testing using ligase chain reaction (LCR) techniques. Analysis of the survey data and in-depth qualitative interviews were undertaken to explore the factors that influenced participants' decisions to participate.

Results: $143 / 199$ (72\%) participants agreed to provide a urine sample. The likelihood of providing a urine sample was reduced if other individuals were present in the home at the time of interview (OR 0.42, 95\% confidence interval $0.20-0.90, \mathrm{p}=0.03$ ). Trust and rapport with the interviewer, understanding the aims of the test, sense of obligation, and perceived importance of the test were identified as additional influencing factors in the in-depth interviews.

Conclusions: Survey respondents' uncertainty or embarrassment at participating in urine testing can be overcome if they are well informed, motivated by the potential health gain, and briefed by trained and confident interviewers.

(Sex Transm Inf 2001;77:194-198)

Keywords: screening; chlamydia; sexually transmitted diseases; survey; sexual behaviour

\section{Background}

Genital chlamydial infection is the most common bacterial sexually transmitted disease in England and Wales with over 40000 cases being diagnosed in genitourinary medicine (GUM) clinics annually. ${ }^{1}$ Concerns regarding the adverse outcomes of untreated infection have led to the recommendation for population screening by the chief medical officer for England. ${ }^{2}$

However, relatively little is known about the prevalence of genital infection with Chlamydia trachomatis in the general population. Community based prevalence studies have been greatly facilitated by the availability of nucleic acid amplification tests that enable non-invasive diagnosis of prevalent infections, ${ }^{3}$ and, to date, only a few of such surveys have been undertaken in the United States and Europe. ${ }^{4-7}$ Prevalence estimates from selected clinic populations (generally among women and involving the use of cervical screening tests) range from $2-12 \% .^{2}{ }^{8}$ Similar estimates among men and sexually active people not accessing health services are lacking.

Incorporating urinary testing for genital chlamydial infection into a community based survey of sexual attitudes and lifestyles provides one method for obtaining prevalence estimates in a representative sample of the general population, of validating reported behaviours, and of identifying demographic and behavioural risk factors for infection. ${ }^{7}$ In this paper we focus on the factors which influence survey participants' willingness to provide a urine sample for chlamydia testing.

\section{Methods}

The feasibility study for an updated probability sample national survey of sexual attitudes and lifestyles (NATSSAL) was undertaken between November 1997 and January 1998..$^{\circ}$ A stratified clustered design was used to identify 3360 addresses in Britain. Half were in London and the remainder spread throughout the rest of Britain in proportion to the population distribution. Overall weighted response to the feasibility survey was $64.7 \%$ of individuals selected in households with a resident aged 16-44 years. A total of 901 completed interviews were obtained. Computer assisted personal interviews on sexual attitudes and behaviours were undertaken by trained interviewers in respondents' homes

As part of this larger feasibility study, randomly selected, sexually experienced respondents from London, aged 18-44 years $(n=199)$, were invited to provide a urine specimen for ligase chain reaction (LCR) testing for $C$ trachomatis infection. Trained interviewers verbally introduced the study at the end of the main interview and provided written information regarding genital chlamydia infection, the rationale for the study and the nature of the urine test. Respondents were informed that they would be only be notified (by letter) in event of a positive LCR result. 
Respondents gave signed informed consent before providing $10 \mathrm{ml}$ of urine in a plastic, preservative-free, sterile container. This was returned to the interviewer who labelled (study identification number and date) and immediately refrigerated the samples at $2-8^{\circ} \mathrm{C}$. Samples were couriered within 24 hours to a central laboratory for LCR testing. On arrival, a $1 \mathrm{ml}$ aliquot of each sample was centrifuged and processed according to the manufacturer's (Abbot LCx Chlamydia trachomatis assay) instructions. Positive and equivocal LCR results were reported to the study physician who then notified respondents.

All patients diagnosed $C$ trachomatis positive or equivocal by LCR were contacted by post. The first letter informed respondents of the possibility of infection and invited them to contact the study physician to discuss their results. If necessary, reminder letters were sent after 7,14 , and 21 days. On contacting the study physician, respondents were told about their test result, options for treatment, and the need for partner notification. Retesting at local sites was only advocated for those receiving equivocal tests. Respondents gave consent for results to be passed on to their general practitioner or local GUM clinic, and to be recontacted after 2 weeks to follow up treatment and partner notification outcomes.

QUANTITATIVE DATA COLLECTION AND ANALYSIS Statistical analysis was performed using the survey analysis functions of STATA version 6 , which incorporates the design effects of clustering of addresses within sectors. Demographic, sexual behaviour (including number of partners, sexual practices, condom use), and STD history (including previous history, treatment site) data were asked of all study participants (see table w1 on STI website). Among those invited to participate in the chlamydia study, we compared those who provided a urine sample with those who did not, to explore what factors influenced participation. When the data were ordinal - for example, number of partners in the past year, the actual numbers reported were used to calculate the overall $\mathrm{p}$ value, rather than the grouped information presented in the tables. Unadjusted odds ratios and their confidence intervals were calculated using the logistic regression function, taking urine provision as the dependent variable. A range of process measures was also collected on each diagnosed case, including time to result notification, time to contact the chlamydia study team, number of notifications required, treatment site preference, and partner notification outcomes.

QUALITATIVE DATA COLLECTION AND ANALYSIS Post-interview, a sample of respondents was approached to participate in in-depth qualitative interviews to explore their experience of taking part in the survey. Thirty six respondents were purposely selected on a range of criteria including age, sex, marital status, and participation in the chlamydia study. All were recruited by telephone. The tape recorded interviews were conducted in respondents' homes by specialist interviewers and transcribed verbatim. Participants ranged from 18-44 years in age, with equal numbers of men and women. Their range of lifetime partners was 4-150 (respondents with fewer than four partners were excluded as the qualitative study also explored how respondents counted partners). Interview transcripts were analysed using Framework (an analytic tool which facilitates systematic within and across case analysis) ${ }^{10}{ }^{11}$ by two independent researchers.

ETHICS

The study was approved by the joint UCL/ UCLH committees on the ethics of human research. The researchers restricted study participation to those aged 18 years and over, following concerns about compromising the confidentiality of those aged 16 and 17 years, for whom parental consent would be required.

\section{Results}

OUTCOMES OF URINARY GENITAL CHLAMYDIAL

INFECTION TESTING

One hundred and forty three (72\%) of the 199 selected London respondents agreed to participate in the chlamydia study. Response was slightly higher among women than men $(73 \% v$ $65 \%, p=0.27$ ). Four men and 12 women tested LCR positive or equivocal for $C$ trachomatis; median (range) ages of 27.5 (18-38) years and 33.5 (22-40) years respectively. Most respondents were notified within 3 weeks of being screened (median 21 days, range 9-32). Once notified by post, $14(88 \%)$ respondents contacted the study physician after receiving their first notification letter. The median time taken by respondents to contact the study team was 2.5 days (range $2-33$ days). Seven respondents chose to be treated by their general practitioners and eight were referred to their local STD clinic. With the exception of one respondent who elected to be treated homeopathically, all respondents received appropriate treatment from a medical practitioner. Partner notification was undertaken with all respondents seen in the GUM clinic, and with four of five respondents seen at GP surgeries for whom information was obtained.

FACTORS INFLUENCING THE PROVISION OF A URINE SAMPLE

A range of demographic and behavioural variables was used to explore the factors associated with providing a urine sample. Despite some suggestion of lower participation rates among younger people, ethnic minorities, and those with lower educational achievement, the presence of "anyone else in the house at the time" (odds ratio $0.42,95 \%$ confidence interval $0.20-0.90, p=0.03$ ) was the only factor found to be significantly associated with a reduced likelihood of participation.

QUALITATIVE RESULTS

The qualitative study shed light on other factors that influenced participation in the chlamydia study. These included the individual's motivation to participate; trust and rapport with the interviewer; understanding of the aims 
of the test and what would happen to samples; a sense of obligation; lack of embarrassment; perceived importance of the test; and the opportunity to receive free testing.

Motivation for participating

For some respondents, agreeing to provide a urine sample was simply "no big deal," while others recognised the importance of collecting data on chlamydia infection. Respondents also valued the opportunity to receive what was regarded as a free screening test. Less positively, there was a sense of obligation and resigned agreement on the premise that the urine test could be no more embarrassing or personal than the survey.

(A) How did you feel when you were asked [to provide a urine sample]?

I was quite surprised. I was very happy to do it because I think it's a brilliant thing and the fact that the lady told me that if there were any problems I would be contacted . . .I think that if you can get any screening done, no matter how, it's a good idea. (Female, aged 35-39 years)

(B) How did you feel when she first asked for a urine sample?

I suppose, yes, I was a bit apprehensive, "what does she want my urine for?" . . but obviously I was thinking about it.

What made you finally decide to give [a sample]? I'd given all my private secrets out so why not give the urine? (Female, aged $40-44$ years)

Level of trust and rapport with interviewer

Most respondents felt the practicalities of the test were handled competently and sensitively by the interviewers and did not find the situation problematic. Despite this, some felt rather embarrassed at giving their sample to a complete stranger to "someone off the street." Had the interviewers been medical personnel this awkwardness may have been relieved to some degree.

(A) It's a medical thing isn't it really? It should be done by doctors or nurses and not someone who comes in your front door. (Male aged 35-40 years)

(B) What factors made you agree to produce a urine sample?

Confidence in the interviewer, very much so. But for that rapport that we had, it would have been much more of a consideration. (Male, aged 30-34 years)

Understanding the aims of the test

Despite interviewer reassurance, there was concern that the sample would be used for purposes other than those stated by the research team. For instance, the misconception that the test constituted a free health check led one man to refuse because he had recently had a health check and did not see the need for another one. Suspicions concerning the use of samples engendered reluctance and, on occasion, refusal to take part. Again the information given by the interviewer is crucial.

(A) .. .I just felt uncomfortable 'cos it's going to a research institution. I don't necessarily trust research teams. They could say they are testing for one thing but really they could be testing for something else. (Female, aged 20-24 years)

Timing of test

The chlamydia study was introduced to the respondents at the end of the interview. For some, the timing of this introduction and explanation was not a problem-others were taken aback by this rather unexpected announcement and said they would have preferred an explanation at the beginning. Competent and reassuring explanations given by the interviewer generally assuaged feelings of being misled, but refusal was not always avoided at this stage. Among those preferring an explanation at the beginning, some admitted that they would have been concerned about the test during the interview and might have even refused to do the whole survey. Interviewers who put forward persuasive arguments and explain the test clearly seemed to be able to mitigate the effects of a last minute explanation.

Attitudes towards being notified of a positive test result

After providing a sample in the survey, concerns about the test continued to stem from the suspicion about how the sample would be used rather than whether the result would be positive. Respondents were generally interested in the result and those who were slightly concerned tended to pay careful attention to phone calls and letters over the following weeks. While some respondents were happy to be informed only in event of a positive result, other preferred to be informed regardless of whether the result was positive or negative.

(A) Did [the result] worry you?

No, not really. I did think at one stage, "what have I got myself into?" and then after that it didn't worry me, no. (Female, aged 35-39 years)

(B) How did you feel about not being told [unless the result is positive]

I don't mind about that 'cos if there isn't anything in it then it doesn't bother me. I know that if the phone don't ring then I haven't [got it]. (Laughs) (Female, aged 35-39 years)

The research highlighted the risk that respondents who have misunderstood the purpose of the test may assume that a negative test result extends to all STDs, thus giving false reassurance.

\section{Discussion}

This is, to our knowledge, the first time that LCR testing for $C$ trachomatis has been incorporated into a general population survey of sexual attitudes and lifestyles in Britain. The response rate $(72 \%)$ was encouraging and points to the feasibility of undertaking urine sampling as part of surveys of this nature. We are also encouraged that respondents did not feel coerced to provide the sample, and generally understood the rationale and relevance of the test. 
Participation bias is a potential problem in community based screening for STDs, in that people who perceive themselves at increased risk may be more (or less) likely to avail themselves of the opportunity for screening. This bias is further compounded when screening is done as part of a survey of sexual attitudes and lifestyles, where survey participants may be more likely to report high risk sexual lifestyles. ${ }^{12}$ The presence of another individual in the household was the only factor (in the quantitative analysis) significantly associated with the decision not to provide a urine sample. However, with a small sample size, we have low power to detect differences in response related to behavioural variables. This will therefore be examined in greater detail in the main survey.

In general, urine testing was acceptable to participants despite having invited respondents to participate only after having completed the main questionnaire. This timing was agreed so as to avoid jeopardising participation to the main study. This practice of sequential informed consent for multiple component surveys has been used previously in other surveys were biological samples are collected. ${ }^{13} 14$ Despite the relatively short notice, we were reassured that respondents did not feel coerced into participating. Qualitative interviews suggested that the attitudes and professionalism of the interviewers and the authority of the researchers and research project offset the anticipated embarrassment and uncertainty.

The benefits of incorporating biological end points in a population based survey of sexual lifestyles are many. We will obtain a greater

\section{Strategies for encouraging participation in urinary testing} for sexually transmitted infections in general population surveys

Respondent strategies

- Provide clear information on Chlamydia trachomatis infection (information sheet, health promotion leaflets, and resources)

- Provide clear written and verbal instructions for how respondents should provide the sample. These should be handled in a professional and factual manner.

- Provide opaque containers for collecting urine specimens

- Inform, agree, and reiterate methods of notifying respondents about results

- Emphasise that a negative result does not provide a clean bill of health for all sexually transmitted infections. Consultation with their nearest genitourinary medicine clinic should be advised.

Interviewer strategies

- Ensure that interviews are well informed about genital chlamydia infection, its outcomes if untreated and the rationale for testing

- Simplify procedures for collecting, labelling, and transporting samples

- Ensure that procedures understood and agreed by interviewers

- Explore interviewer's health and hygiene concerns and try to resolve where possible (reassurance, provision of gloves to handle specimen containers, refrigeration facilities)

- Provide feedback and encouragement on progress of recruitment and recruitment strategies. understanding of the epidemiology of $C$ trachomatis and its association with sexual behaviours, and a range of demographic factors, which will help guide screening strategies. As the study is a population based probability sample, many of the biases associated with recruiting from sexual or reproductive health clinics are avoided, and more robust population based prevalence estimates can be obtained.

The results of the feasibility study are encouraging and will inform the development of future large scale surveys of this nature. $\mathrm{Ku}$ and colleagues ${ }^{7}$ have, however, outlined some potential challenges to integrating behavioural and clinical data in population based surveys. Key among these are close collaboration between clinicians and survey researchers, flexibility of survey methods to optimise participation rates, and dealing effectively with ethical issues. This study highlighted more pragmatic considerations to ensure the success of community based testing for STIs (see box). Adequate investment must be placed in training interviewers, particularly about the sexually transmitted infection being examined, and simplifying the process of collecting and transporting samples. For respondents, professional and relaxed interviewers, who focus on the benefits of testing and utilise a non-coercive approach are key. The acceptability of urine testing may also be improved by providing clear information and guidance on the project. Interviewers should also make it clear at the time of recruitment that a negative result or no feedback is not a comprehensive clean bill of sexual health, and this should be reiterated in the information leaflets. This study provides justification for utilising a broadly similar approach in the 1999/2000 Second National Survey of Sexual Attitudes and Lifestyles (NATSSAL 2), ${ }^{15}$ currently in progress.

\section{Conflict of interest: None.}

The study was funded by a grant from the Medical Research Council. We thank the team of interviewers of the National Centre for Social Research (formerly Social and Community Planning Research) and the participants for their contributions. We also acknowledge the significant contribution of Jane We also acknowledge the significant contribution of Jane of this project. We would like to thank Sundhiya Mandalia for preliminary analyses undertaken on the dataset.

Sunced Contributors: KF was the study physician who managed the study and was the lead writer of the paper; AJ, JF, and KW were principal investigators, who along with $\mathrm{KF}$ and $\mathrm{BE}$ developed the methodology for the chlamydia study and questionnaire design; AC is the study statistician and undertook the statistical analysis presented in this paper; CC and GR were microbiological laboratory collaborators who undertook the LCR testing and provided comments on the draft; KM, GE, and KW designed and undertook the qualitative interviewing and contributed to the paper writing.

1 Lamagni TL, Hughes G, Rogers PA, et al. New cases seen at genitourinary clinics: England 1998. CDR Suppl.

2 Chief Medical Officer's Expert Advisory Group. Main report of the CMO's Expert Advisory Group on Chlamydia trachomatis. London: Department of Health, 1998.

3 Black C. Current methods of laboratory diagnosis of Chlamydia trachomatis infections. Clin Microbiol Rev 1997; 10:160-84.

4 Grun L, Tassano-Smith J, Carder C, et al. Comparison of two methods of screening for genital chlamydial infection in women attending in general practice: cross sectional survey. BMF 1997;315:226-30.

5 Ostergaard L, Andersen B, Olesen F, et al. Efficacy of home sampling for screening of Chlamydia trachomatis: ransampling for screening of Chlamydia

6 Macleod J, Rowsell R, Horner P, et al. Postal urine specimens: are they a feasible method for genital chlamydial infection screening? Br f Gen Pract 1999;49:455-8. 
$7 \mathrm{Ku}$ L, Sonenstein FL, Turner CF, et al. The promise of integrated representative surveys about sexually transmitted
diseases and behaviour. Sex Transm Dis 1997;24:299-309.

8 Stokes T. Screening for Chlamydia in general practice. A literature review and summary of the evidence. $f$ Public Health Med 1997;19:222-32.

9 Johnson AM, Copas AJ, Erens B, et al. Effect of computer assisted self-interviews on reporting of sexual HIV risk behaviours in a general population sample. A methodological experiment. AIDS (in press).

10 Bryman A, Burgess RG. Analysing qualitative data. London: Routledge, 1994:173-94.

11 Mitchell K, Wellings K, Elam G. NATSSAL 2: Survey design and implementation, report of the qualitative stage.
London: London School of Hygiene and Tropical Medicine, 1998

12 Copas A, Johnson AM, Wadsworth J. Assessing participation bias in a sexual behaviour survey: implications for measuring HIV risk. AIDS 1997;11:783-90.

13 Prescott-Clarke P, Primatesta P, eds. Health survey for England, the health of young people '95-97. Vols 1 and 2. London: Stationery Office, 1998

14 Finch S, Doyle W, Lowe C, et al. National diet and nutrition survey: people aged 65 years and over. Vols 1 and 2. London: Stationery Office, 1998.

15 Department of Health. Department of Health to fund survey 1998/0468: 26 October 1998. 